\title{
Blood kinetics of Ebola virus in survivors and nonsurvivors
}

\author{
Simone Lanini, ${ }^{1}$ Gina Portella, ${ }^{2}$ Francesco Vairo, ${ }^{1}$ Gary P. Kobinger, ${ }^{3}$ Antonio Pesenti, ${ }^{2,4}$ Martin Langer, ${ }^{2,5}$ Soccoh Kabia, ${ }^{6}$ \\ Giorgio Brogiato, ${ }^{2}$ Jackson Amone, ${ }^{7}$ Concetta Castilletti, ${ }^{1}$ Rossella Miccio, ${ }^{2}$ Alimuddin Zumla, ${ }^{8}$ Maria Rosaria Capobianchi, ${ }^{1}$ \\ Antonino Di Caro, ${ }^{1}$ Gino Strada, ${ }^{2}$ Giuseppe Ippolito, ${ }^{1}$ and INMI-EMERGENCY EBOV Sierra Leone Study group ${ }^{9}$

\begin{abstract}
'National Institute for Infectious Diseases “Lazzaro Spallanzani”, Rome, Italy. ${ }^{2}$ EMERGENCY NGO, Milan, Italy. ${ }^{3}$ Special Pathogens Program, National Microbiology Laboratory, Public Health Agency of Canada, Winnipeg, Manitoba, Canada. " ${ }^{4}$ Department of Health Science, University of Milan-Bicocca, and Department of Emergency and Intensive Care, San Cerardo Hospital, Monza, Italy. ${ }^{5}$ Department of Pathophysiology and Transplantation, University of Milan, and Fondazione IRCCS Istituto Nazionale dei Tumori, Milan, Italy. ${ }^{6}$ Connaught Hospital Tower Hill, Freetown, Sierra Leone. 'Department of Clinical Services, Ministry of Health, Kampala, Uganda. ${ }^{8}$ Division of Infection and Immunity, University College London, and NIHR Biomedical Research Centre at UCL Hospitals NHS Foundation Trust, London, United Kingdom. ${ }^{9}$ The collaborative group is detailed in the Supplemental Material.
\end{abstract}

BACKGROUND. Infection with Ebola virus (EBOV) results in a life-threatening disease, with reported mortality rates between $50 \%-70 \%$. The factors that determine patient survival are poorly understood; however, clinical observations indicate that EBOV viremia may be associated with fatal outcome. We conducted a study of the kinetics of Zaire EBOV viremia in patients with EBOV disease (EVD) who were managed at an Ebola Treatment Centre in Sierra Leone during the recent West African outbreak.

METHODS. Data from 84 EVD patients (38 survivors, 46 nonsurvivors) were analyzed, and EBOV viremia was quantified between 2 and 13 days after symptom onset. Time since symptom onset and clinical outcome were used as independent variables to compare EBOV viral kinetics in survivors and nonsurvivors.

RESULTS. In all patients, EBOV viremia kinetics was a quadratic function of time; however, EBOV viremia was 0.94 logarithm $(\mathrm{log})$ copies per $\mathrm{ml}(\mathrm{cp} / \mathrm{ml})(P=0.011)$ higher in nonsurvivors than in survivors from day 2 after the onset of symptoms. Survivors reached peak viremia levels at an earlier time after symptom onset than nonsurvivors (day 5 versus day 7) and had lower mean peak viremia levels compared with nonsurvivors (7.46 log cp/ml; 95\% Cl, 7.17-7.76 vs. $8.60 \log \mathrm{cp} / \mathrm{ml}$; $95 \% \mathrm{Cl}$, 8.27-8.93). Before reaching peak values, EBOV viremia similarly increased both in survivors and nonsurvivors; however, the decay of viremia after the peak was much stronger in survivors than in nonsurvivors.

CONCLUSION. Our results demonstrate that plasma concentrations of EBOV are markedly different between survivors and nonsurvivors at very early time points after symptom onset and may be predicative of outcome. Further studies focused on the early phase of the disease will be required to identify the causal and prognostic factors that determine patient outcome.

FUNDING. Italian Ministry of Health; Italian Ministry of Foreign Affairs; EMERGENCY's private donations; and Royal Engineers for DFID-UK.

\section{Introduction}

Ebola virus (EBOV) is one of the most deadly viral infections of humans, with mortality rates being reported between $50 \%-70 \%$. The recent EBOV disease (EVD) epidemic in Western Africa presented huge challenges for public health and for conducting basic science and translational clinical research $(1,2)$. As of September 13,2015 , WHO estimated that there were 28,265 cases of EVD, with 11,308 deaths associated with the recent West African outbreak (3).

Clinical observations show that patients with EVD who did not survive showed severe symptoms and clinical signs during the early phase of the disease; many progressed to developing multiorgan failure, resulting in death by the end of the second week. Patients

Conflict of interest: The authors have declared that no conflict of interest exists. Submitted: June 8, 2015; Accepted: September 28, 2015.

Reference information: J Clin Invest. 2015;125(12):4692-4698. doi:10.1172/JCI83111. who survived typically began to improve during the second week of illness. Performing clinical, laboratory, and pathogenesis studies during EVD outbreaks is extremely difficult due to biosafety, and logistical and operational issues. EBOV viral load in blood, or viremia, may reflect the dynamic interaction between viral replication and clearance by the body's immune system. While recent studies have suggested that EBOV viremia may be a predictor of clinical outcome $(4,5)$, no accurate data are available on the temporal trends of kinetics of EBOV viremia during the course of the disease.

We conducted a study of the kinetics of EBOV viremia in patients with EVD who were managed in an Ebola Treatment Centre (ETC) in Goderich-Freetown, Sierra Leone. To obtain optimal information contained in datasets composed of sparse repeated measures, we used an inferential approach that has been recently applied to clinical infectious diseases (6) and microbial ecology (7). 


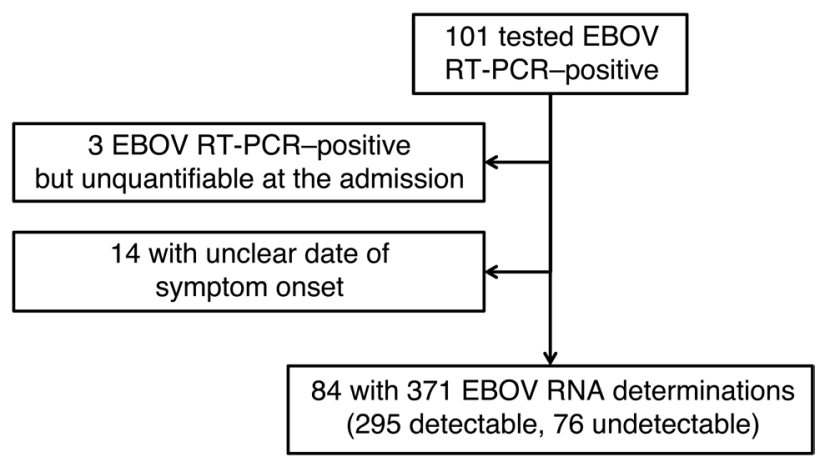

\section{Results}

Descriptive analysis. Between December 13 ,2014, and April 20, 2015 , a total of 101 patients with laboratory-confirmed diagnosis of EVD were admitted to the EMERGENCY NGO ETC in Goderich-Freetown, Sierra Leone. Of 101 patients, there were 50 survivors and 51 nonsurvivors (50.5\% mortality). Selection of patients for this is depicted in Figure 1. Table 1 shows the age groups, sex, and clinical characteristics of 84 patients (38 survivors and 46 nonsurvivors) that were studied for analysis. The mean time between onset of symptoms and admission was 4.53 days (SD 2.48). In patients who did not survive, the mean time between onset of symptoms and death was 9.04 days (SD 3.17). The mean time between onset of symptoms and first undetectable EBOV viremia in survivors was 12.71 days (SD 3.84).
Figure 1. Selection of patients. Between December 13, 2014, and April 20, 2015, 101 patients tested positive to EBOV RT-PCR, of whom 84 were included in the analysis. For these 84 patients, a total of 295 measurable EBOV viremia results were available and 76 had undetectable EBOV RNA.

\section{Table 1. Bivariable analysis of the patients included in the analysis}

$\begin{array}{lll}\text { Subjects } & & \\ \text { Feature } & & \text { No } \\ \text { Sex } & \text { Female } & 44 \\ & \text { Male } & 40 \\ \text { Age } & 0-19 & 20 \\ & 20-39 & 42 \\ & \geq 40 & 22 \\ \text { Malaria } & \text { Neg. } & 74 \\ & \text { Pos. } & 10 \\ \text { Drugs }{ }^{\complement} & \text { None } & 60 \\ & \text { Amiodarone } & 19 \\ & \text { MAbs } & 19 \\ \text { Admission } & & 5 \\ & <5 \text { days } & 42 \\ \text { Outcome } & \geq 5 \text { days } & 42 \\ & \text { Survivors } & 38 \\ \text { Mechanical ventilation } & \text { Nonsurvivors } & 46 \\ & \text { No } & 56 \\ & \geq 1 \text { day } & 28 \\ \text { Renal replacement } & \text { No } & 66 \\ & \geq 1 \text { day } & 18 \\ \text { Overall } & & 84 \\ & & \end{array}$

$\begin{array}{cc} & \text { EBOV viremia } \\ \text { No. } & \text { Mean (95\% CI) } \\ 44 & 7.72(7.34-8.10) \\ 40 & 7.88(7.48-8.28) \\ 20 & 8.17(7.61-8.73) \\ 42 & 7.67(7.28-8.06) \\ 22 & 7.69(7.16-8.23) \\ 74 & 7.74(7.45-8.03) \\ 10 & 8.19(7.40-8.99) \\ 60 & 7.89(7.56-8.21) \\ 19 & 7.47(6.89-8.05) \\ 5 & 7.93(6.77-9.03) \\ 42 & 7.74(7.35-8.13) \\ 42 & 7.85(7.46-8.24) \\ 38 & 7.11(6.75-7.46) \\ 46 & 8.36(8.03-8.68) \\ 56 & 7.54(7.22-7.86) \\ 28 & 8.31(7.85-8.77) \\ 66 & 7.66(7.35-9.97) \\ 18 & 8.25(7.70-8.87) \\ 84 & 7.80(7.52-8.07)\end{array}$

\begin{tabular}{|c|c|}
\hline Difference $^{A}(95 \% \mathrm{Cl})$ & $p^{\mathrm{B}}$ \\
\hline $\begin{array}{c}\text { Base } \\
+0.16(-0.39 \text { to }+0.71)\end{array}$ & 0.567 \\
\hline $\begin{array}{c}\text { Base } \\
-0.50(-1.18 \text { to }+0.19) \\
-0.48(-1.25 \text { to }+0.30)\end{array}$ & 0.326 \\
\hline $\begin{array}{c}\text { Base } \\
+0.45(-0.40 \text { to }+1.30)\end{array}$ & 0.295 \\
\hline $\begin{array}{c}\text { Base } \\
-0.42(-1.08 \text { to }+0.24) \\
+0.14(-1.16 \text { to }+1.19)\end{array}$ & 0.450 \\
\hline $\begin{array}{c}\text { Base } \\
+0.11(-0.44 \text { to }+0.66)\end{array}$ & 0.695 \\
\hline $\begin{array}{c}\text { Base } \\
+1.25(+0.76 \text { to }+1.73)\end{array}$ & $<0.001$ \\
\hline $\begin{array}{c}\text { Base } \\
+0.77(+0.21 \text { to }+1.33)\end{array}$ & $<0.00$ \\
\hline $\begin{array}{c}\text { Base } \\
+0.62(-0.04 \text { to }+1.28)\end{array}$ & 0.063 \\
\hline
\end{tabular}

\begin{tabular}{|c|c|c|}
\hline \multicolumn{3}{|c|}{ Highest EBOV viremia during hospital stay ( $\log \mathrm{cp} / \mathrm{ml})$} \\
\hline Mean $(95 \% \mathrm{Cl})$ & Difference ${ }^{A}(95 \% \mathrm{Cl})$ & $P^{\mathrm{B}}$ \\
\hline 7.92 (7.52 to 8.32 ) & Base & 0.206 \\
\hline $8.29(7.86-8.70)$ & $+0.37(-0.21$ to +0.94$)$ & \\
\hline $8.44(7.85-9.03)$ & Base & \\
\hline $7.99(7.58-8.40)$ & $-0.45(-1.17$ to +0.27$)$ & 0.414 \\
\hline $7.97(7.40-8.53)$ & $-0.47(+1.29$ to -0.35$)$ & \\
\hline $8.02(7.71-8.32)$ & Base & 0.173 \\
\hline $8.63(7.80-9.46)$ & $+0.61(-0.27$ to +1.50$)$ & \\
\hline $8.05(7.71-8.39)$ & Base & 0.446 \\
\hline $8.03(7.41-8.63)$ & $-0.03(-0.72$ to +0.67$)$ & \\
\hline $8.82(7.64-10.01)$ & $+0.77(-0.45$ to +2.01$)$ & \\
\hline $8.15(7.74-8.56)$ & Base & 0.686 \\
\hline $8.03(7.62-8.44)$ & $-0.12(-0.70$ to +0.45$)$ & \\
\hline $7.28(6.92-7.64)$ & Base & $<0.001$ \\
\hline $8.75(8.43-9.08)$ & $+1.47(+0.99$ to 1.96$)$ & \\
\hline $7.73(7.40-8.06)$ & Base & \\
\hline $8.81(8.34-9.28)$ & $+1.08(+0.51$ to +1.65$)$ & $<0.001$ \\
\hline $7.89(7.58-8.20)$ & Base & \\
\hline $8.82(8.22-9.42)$ & $+0.93(+0.25$ to +1.60$)$ & 0.008 \\
\hline $8.09(7.80-8.38)$ & - & - \\
\hline
\end{tabular}

HIV Ab, test for anti-HIV antibodies; MAbs, anti-EBOV monoclonal antibodies. ${ }^{A}$ Difference of mean EBOV viremia in log cp/ml in comparison to base level; ${ }^{B} P$ value according to standard linear regression model ( $F$ test); ${ }^{C} d r u g$ with potential effect on EBOV viremia; ${ }^{D}$ amiodarone was administered at admission (day 0) as $5 \mathrm{mg} / \mathrm{kg}$ in 1 hour i.v. Between days 1-3, patient received amiodarone i.v. $20 \mathrm{mg} / \mathrm{kg} / 24$ hours (continuous infusion); between days 4-10, patients received amiodarone per os $10 \mathrm{mg} / \mathrm{kg}$ every 8 hours. EOne patient received ZMAb; 1 patient received ZMAb in addition to amiodarone; 2 patients received ZMAPP; 1 patient received ZMAPP in addition to amiodarone. FTime between symptom onset and hospital admission. 


\section{Table 2. Multivariable analysis of the patients included in the analysis}

\section{Subjects}

Feature

$\begin{array}{lll}\text { Outcome } & \text { Survivors } & 38 \\ & \text { Nonsurvivors } & 46 \\ \text { Mechanical ventilation } & \text { No } & 56 \\ & \geq 1 \text { day } & 28 \\ \text { Renal replacement } & \text { No } & 66 \\ & \geq 1 \text { day } & 18\end{array}$

EBOV viremia at admission $(\log \mathrm{cp} / \mathrm{ml})$

$\begin{array}{ccc}\begin{array}{c}\text { Adjusted mean } \\ (95 \% \mathrm{Cl})\end{array} & \begin{array}{c}\text { Adjusted difference } \\ (95 \% \mathrm{Cl})\end{array} & P^{A} \\ 7.15(6.76-7.54) & \text { Base } & <0.001 \\ 8.33(7.97-8.68) & +1.17(+0.61 \text { to }+1.73) & \\ 7.74(7.40-8.08) & \text { Base } & \\ 7.90(7.38-8.43) & +0.16(-0.54 \text { to }+0.86) & 0.649 \\ 7.80(7.50-8.08) & \text { Base } & \\ 7.80(7.15-8.44) & -0.00(-0.75 \text { to }+0.75) & 1.00\end{array}$

\begin{tabular}{|c|c|c|}
\hline \multicolumn{3}{|c|}{ Highest EBOV viremia during hospital stay $(\log \mathrm{cp} / \mathrm{ml})$} \\
\hline $\begin{array}{l}\text { Adjusted mean } \\
(95 \% \mathrm{Cl})\end{array}$ & $\begin{array}{l}\text { Adjusted difference } \\
\qquad(95 \% \mathrm{Cl})\end{array}$ & $P^{A}$ \\
\hline $7.40(7.01-7.77)$ & Base & $<0.001$ \\
\hline $8.67(8.32-9.02)$ & $1.27(+0.71$ to 1.83$)$ & \\
\hline $7.97(7.63-8.31)$ & Base & \\
\hline $8.33(7.80-8.85)$ & $+0.35(-0.34$ to +1.05$)$ & 0.316 \\
\hline $8.01(7.77-8.36)$ & Base & \\
\hline $8.19(7.55-8.83)$ & $+0.12(-0.62$ to +0.87$)$ & 0.745 \\
\hline
\end{tabular}

Log cp/ml, decimal log of EBOV RNA copies per milliliter. AValue according to standard multivariable linear regression model ( $F$ test).

Multivariable analyses showed no significant association between EBOV viremia at admission and the need for mechanical ventilation $(P=0.649)$ or the need for renal replacement $(P=1.00)$. Similarly, there was no significant association between the mean of the highest viremia values during hospitalization and the need for mechanical ventilation $(P=0.316)$ or the need for renal replacement $(P=0.745)$.

Modeling the kinetics of EBOV viremia. Temporal trends of EBOV viremia levels for the 84 patients were modeled using 275 quantitative PCR (qPCR) results (3.3 determinations per patient) between days 2 and 13 after first symptom onset. This range was selected in order to have at least 10 real observations for each time point estimate, and it led to the exclusion of 20 EBOV viremia determinations between days 14 and 17. The final model included the random intercept at patient level, the random slope at time level, EBOV viremia as a dependent variable and 2 independent variables (i.e., time after symptom onset, as a continuous quadratic term), and patient's clinical outcome (as a binary term). According to model-based likelihood ratio tests (LRT), we also included full interaction (i.e., linear and quadratic) between the 2 independent variables. Following the results of multivariable analysis (Table 2), no potential confounder was included. Model details and parameters for fixed and random coefficients are shown in Table 3.

Variation of EBOV viremia over time since symptom onset. Between days 2 and 13 after the onset of symptoms, EBOV viremia in nonsurvivors was always significantly higher than in survivors (Table 4). At day 2 after onset of symptoms (which represents the model intercept), viremia levels were signifi- cantly higher in nonsurvivors compared with survivors (0.94 log $\mathrm{cp} / \mathrm{ml}$ [95\% CI, 0.21-1.67]; $P=0.011)$. This difference increased to $1.50 \log \mathrm{cp} / \mathrm{ml}$ (95\% CI, 0.99-2.01; $P<0.001)$ and $4.94 \log \mathrm{cp} / \mathrm{ml}$ $(95 \%$ CI, 3.63-6.25; $P<0.001)$ at day 7 and day 13 after the onset of symptoms, respectively (Table 4 ).

Survivors reached their viremic peak value earlier than nonsurvivors (day 5 versus day 7 after symptom onset, respectively). The mean peak value of viremia in survivors was lower than in

\section{Table 3. Full model parameters}

\section{Overall model parameters}

Number of observation: RNA determination

275

Random intercept group variable: patients

Random slope variable (time): days

84

Observation per patients: (RT-PCR with detectable RNA levels)

3.3

\section{Model selection}

Random effect (assessed on null model)

Random intercept vs. standard linear regression model $\quad P<0.001$

Random slope vs. random intercept model $\quad P<0.001$

Functional form of association between time and viremia

Linear vs. quadratic $\quad P<0.001$

Quadratic vs. cubic

$P=0.699$

Quadratic vs. $4^{\text {th }}$ power

$P=0.911$

Quadratic vs. $6^{\text {th }}$ power

$P=0.543$

Quadratic vs. categorical 12-level

$P=0.560$

Interaction between time and outcome vs. no interaction

\section{Fixed effect parameters}

Time after symptom onset: linear

Time after symptom onset: quadratic

Clinical outcome: binary (0 survived, 1 died)

Interaction

Time after symptom onset (linear) and clinical outcome

Time after symptom onset (quadratic) and clinical outcome

Constant: Intercept at Time after symptom onset $=2$

\section{Random effect parameters}

Random slope

Random intercept

Unstructured covariance

Residual variance

$P<0.001$

Coeff., coefficient. 
Table 4. Predicted value of EBOV viremia in survivors and nonsurvivors

\begin{tabular}{|c|c|c|c|c|c|}
\hline \multirow[t]{2}{*}{ Days $^{A}$} & \multicolumn{2}{|c|}{ Patients } & \multirow{2}{*}{$\begin{array}{c}\text { EBOV viremia } \\
\log \mathrm{cp} / \mathrm{ml}(95 \% \mathrm{Cl})\end{array}$} & \multicolumn{2}{|c|}{ Difference $^{c}$} \\
\hline & Outcome & No. ${ }^{B}$ & & $\log \mathrm{cp} / \mathrm{ml}(95 \% \mathrm{Cl})$ & $P^{D}$ \\
\hline \multirow[t]{2}{*}{2} & Survivors & 5 & $7.03(6.49-7.58)$ & Base & 0.011 \\
\hline & Nonsurvivors & 9 & $7.97(7.49-8.45)$ & 0.94 (+0.21 to 1.67$)$ & \\
\hline \multirow[t]{2}{*}{3} & Survivors & 5 & $7.31(6.90-7.71)$ & Base & 0.001 \\
\hline & Nonsurvivors & 7 & $8.19(7.83-8.55)$ & $0.88(0.34-1.42)$ & \\
\hline \multirow[t]{2}{*}{4} & Survivors & 6 & $7.45(7.13-7.77)$ & Base & $<0.001$ \\
\hline & Nonsurvivors & 14 & $8.36(8.08-8.65)$ & $0.91(0.48-1.34)$ & \\
\hline \multirow[t]{2}{*}{5} & Survivors & 11 & $7.46(7.17-7.76)$ & Base & $<0.001$ \\
\hline & Nonsurvivors & 17 & $8.49(8.22-8.75)$ & $1.02(0.62-1.42)$ & \\
\hline \multirow[t]{2}{*}{6} & Survivors & 15 & $7.35(7.02-7.68)$ & Base & $<0.001$ \\
\hline & Nonsurvivors & 20 & $8.57(8.28-8.85)$ & $1.22(0.78-1.66)$ & \\
\hline \multirow[t]{2}{*}{7} & Survivors & 13 & 7.10 (6.71-7.49) & Base & $<0.001$ \\
\hline & Nonsurvivors & 16 & $8.60(8.27-8.93)$ & $1.50(0.99-2.01)$ & \\
\hline \multirow[t]{2}{*}{8} & Survivors & 13 & $6.72(6.27-7.18)$ & Base & $<0.001$ \\
\hline & Nonsurvivors & 21 & $8.59(8.21-8.96)$ & $1.86(1.27-2.45)$ & \\
\hline \multirow[t]{2}{*}{9} & Survivors & 12 & $6.22(5.69-6.75)$ & Base & $<0.001$ \\
\hline & Nonsurvivors & 19 & $8.53(8.08-8.97)$ & $2.31(1.62-3.00)$ & \\
\hline \multirow[t]{2}{*}{10} & Survivors & 12 & $5.58(4.97-6.19)$ & Base & $<0.001$ \\
\hline & Nonsurvivors & 16 & $8.42(7.90-8.94)$ & $2.84(2.04-3.64)$ & \\
\hline \multirow[t]{2}{*}{11} & Survivors & 6 & $4.81(4.10-5.52)$ & Base & $<0.001$ \\
\hline & Nonsurvivors & 9 & $8.27(7.65-8.88)$ & $3.46(2.52-4.39)$ & \\
\hline \multirow[t]{2}{*}{12} & Survivors & 8 & $3.91(3.09-4.74)$ & Base & $<0.001$ \\
\hline & Nonsurvivors & 8 & $8.07(7.33-8.81)$ & $4.15(3.05-5.26)$ & \\
\hline \multirow[t]{2}{*}{13} & Survivors & 8 & $2.89(1.92-3.85)$ & Base & $<0.001$ \\
\hline & Nonsurvivors & 5 & $7.82(6.94-8.71)$ & $4.94(3.63-6.25)$ & \\
\hline
\end{tabular}

EBOV viremia values are predicted according to patients' clinical outcomes. Analysis was carried out on 275 EBOV viremia determinations (114 from survivors and 161 from nonsurvivors). Log $\mathrm{cp} / \mathrm{ml}$, decimal log of EBOV RNA copies per milliliter. ${ }^{A}$ Days after symptom onset; ${ }^{B}$ number of patients in the specific day after symptom onset (each patient can have only 1 EBOV RNA determination per day); 'difference of mean EBOV viremia in log $\mathrm{cp} / \mathrm{ml}$ between nonsurvivors and survivors; ${ }^{\mathrm{D}} P$ value according ANOVA-style joint tests.

nonsurvivors (7.46 log cp/ml; 95\% CI, 7.17-7.76 vs. $8.60 \log \mathrm{cp} /$ $\mathrm{ml}$; 95\% CI, 8.27-8.93). Model estimates for the peak values were largely consistent with the observed mean of the highest viremia levels recorded during hospitalization $(7.28$ and $8.75 \mathrm{log} \mathrm{cp} / \mathrm{ml}$ EBOV RNA in survivors and nonsurvivors, respectively; Table 1). Among survivors, EBOV viremia reached the limit of detection (LOD) between day 12 and 13 (Figure 2), coinciding with observed mean time between symptom onset and first undetectable EBOV viremia (i.e., 12.71 days).

In both survivors and nonsurvivors, EBOV viral load increased in the early phase of EVD and started decreasing from day 5 in survivors and from day 7 in nonsurvivors. According to the quadratic function (i.e., inverted u-shaped curve), by day 2 after symptom onset, the increase of EBOV viremia before peak values was similar in survivors and nonsurvivors, as modeled by the nonsignificant interaction between linear coefficient for time and clinical outcome (Table 3). In contrast, after peak values, the reduction of viremia was significantly more pronounced in survivors than in nonsurvivors, as modeled by the significant positive interaction between the negative quadratic coefficient for time and clinical outcome (Table 3).
The overall results of the model are shown in Table 4 and Figure 2.

\section{Discussion}

To our knowledge, this is the first study that has modeled the kinetics of EBOV viremia in patients with EVD. It is also the first to highlight the significant link between the temporal trend of EBOV viremia and its relationship to clinical outcome. There are several notable findings of our study. Firstly, early in the course of EVD, soon after the onset of symptoms, EBOV viral load levels were significantly higher in those patients who died compared with those who survived. Secondly, EVD patients who survived reached their peak viral-load levels earlier than nonsurvivors. The mean time between onset of symptoms and first undetectable EBOV viremia in survivors was 12.71 days (SD 3.84) compared with 9.04 days (SD 3.17) between symptom onset and death in nonsurvivors. Thirdly, EBOV viremia between days 2 and 13 after onset of symptoms was a quadratic function of the time. While EBOV viral load levels similarly increased in both survivors and nonsurvivors, after peak values were reached, viral decay in survivors was much more pronounced than in nonsurvivors. There are several possible reasons for this observation, such as differences in host genetic factors, innate and adaptive immune responses, and underlying risk factors and co-morbidities (8-10). Further studies will be required in any future outbreak to determine the potential contributions of each of these factors.

Our findings confirm the inference from clinical studies in West Africa $(4,5)$ and past EVD outbreaks $(8,9,11-13)$ of the possible link between early EBOV viremia and a fatal outcome in EVD patients. The case fatality rate in Sierra Leone ranged between 33\% and $94 \%$ in EVD patients presenting with an EBOV viremia less than $5 \log \mathrm{cp} /$ $\mathrm{ml}$ and more than $7 \log \mathrm{cp} / \mathrm{ml}$, respectively (5). Our findings also concur with those of Towner and colleagues who studied EVD patients from the outbreak in Sudan. They indicated that peak viremia in survivors was observed about 2 days earlier than in nonsurvivors. In addition, despite the potential differences in EBOV strains and the RT-PCR assays, the observed mean values of peak viremia for either survivors or nonsurvivors were comparable to that estimated in our study (12).

Our data is relevant to EVD patients in the West Africa setting. Despite the best available level of supportive care provided in the EMERGENCY ETC in Goderich-Freetown, Sierra Leone, the mortality rate was high, at $>40 \%$. This was twice as high when compared with the mortality rate for the $26 \mathrm{EVD}$ patients who received healthcare in Europe $(14,15)$ and North America $(14,16)$, of whom 5 died (20\% mortality) (17). While the majority of patients with the lowest EBOV viremia levels cleared the virus within the time frame estimated by our model (18), several patients had presented with very high EBOV viremia levels on admission but still went on 

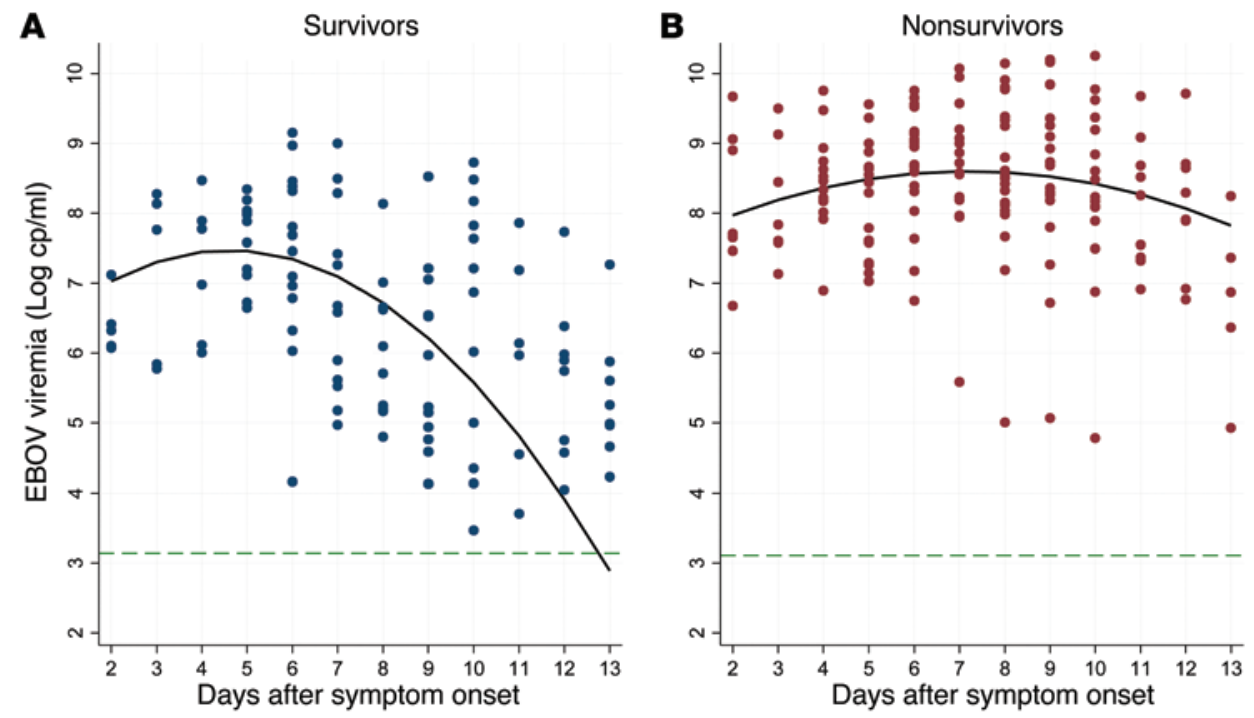

Figure 2. EBOV kinetics. Linear prediction of EBOV RNA blood levels (viremia) according to the time since symptom onset and patients' clinical outcomes. All estimates were made on the full dataset, including 275 EBOV RNA determinations on the 84 patients. (A) Survivors: 38 patients with 114 EBOV RNA determinations. (B) Nonsurvivors: 46 patients with 161 EBOV RNA determinations. Dots indicate single EBOV RNA determination; black line indicates linear prediction according to linear multilevel model; green dashed line indicates the LOD of the EBOV RNA assay (3.11 log cp/ml).

to survive $(19,20)$. This might be attributed to different levels of healthcare and the use of investigational antiviral treatments or differences in innate and acquired immune responses. It is also probable that these differences between the patients in our study and those who received therapy in Europe and North America are completely spurious because of the random error due to small numbers of observations.

Our study data was collected in real time during the ongoing EVD outbreak in West Africa, and thus, our results should be viewed in light of several limitations. Firstly, as nonsurvivors had EBOV RNA levels above $9 \log \mathrm{cp} / \mathrm{ml}$ (i.e., beyond the estimated dynamic range of the test) more frequently than survivors, the model may have underestimated the difference between groups. Secondly, our study may have selection bias; we studied only patients who were admitted to hospital care and did not include patients at the end of both spectrums of EVD who did not seek or receive healthcare - those with fulminant disease or those with very mild illness. Thirdly, since multiple imputation techniques can be unreliable in multilevel models with random slope (21), we handled missing data with a list-wise deletion approach that might have introduced a selection bias. Fourthly, our study provides no statistically significant evidence of association between a patient's baseline condition - such as age, sex, malaria coinfection, and use of drugs - with potential antiviral effect. We performed a sensitivity analysis (data not shown) and found that including patients who received amiodarone or monoclonal antibodies had no effect on the estimation of the coefficients of the multilevel model. Finally, given our study sample size and the real-time exploratory nature of our study, the model cannot be used to make predictions about EBOV viremia kinetics before day 2 and after day 13 since symptom onset.

In conclusion, plasma concentrations of EBOV are significantly different between survivors and nonsurvivors beginning very early after symptom onset and may indicate prognostic significance. Further studies focusing on the early phase of the disease are required to identify prognostic factors that determine patients' outcomes.

\section{Methods}

Study design, setting, and patient followup. We conducted a study of consecutive patients with laboratory-confirmed EVD who were admitted for care in an Ebola treatment center (ETC) in Goderich-Freetown (Sierra Leone), managed by the nongovernmental organization EMERGENCY (22). The ETC is endowed with an internal laboratory for molecular diagnosis of EVD, managed by Italian National Institute for Infectious Diseases "Lazzaro Spallanzani."

Patients were followed up from the day of admission until the day of discharge.

Eligible patients and enrollment criteria. All patients with EVD who were admitted to ETC between December 13, 2014, and April 20, 2015, were retrospectively enrolled in the study. Enrollment was restricted to those who had the time of onset of symptoms recorded and at least one quantifiable blood EBOV RNA result was available.

All EVD suspect cases (according to WHO definition; ref. 23) underwent quantitative EBOV RT-PCR on plasma samples; those who tested positive received multiple EBOV RT-PCR tests during the hospital stay, as clinically indicated. Confirmed EVD cases were discharged after 2 consecutive undetectable EBOV RNA RT-PCRs in blood taken at least 2 days apart. Patients were treated with supportive care according to a standardized protocol (including hemodynamic support, mechanical ventilation, and continuous renal replacement where necessary) developed by EMERGENCY NGO and approved by the Sierra Leone Ministry of Health. Some patients also received drugs with potential antiviral effect, on a case-by-case basis.

Variables. Viremia (as a continuous variable), reported as plasma concentration of EBOV RNA in the decimal log of $\mathrm{cp} / \mathrm{ml}$, was the dependent variable in all inferential models.

Patient's clinical outcome (as a binary variable) and time since symptom onset (as a continuous variable) were used as the 2 a priori independent variables for the multilevel model. Eight additional variables were considered as potential confounders: age (as a categorical variable), sex (as a binary variable), HIV test result (as a binary variable), malaria rapid test result (as a binary variable), receipt of drugs with any potential effect on EBOV viremia (as a categorical variable), need for mechanical ventilation (as a binary variable), need for renal-replacement therapy (as a binary variable), and time between symptom onset and hospital admission (as a binary variable). 
Information regarding day of hospital admission, day of death in nonsurvivors, day of first undetectable EBOV RT-PCR in survivors, and day of discharge was also collected.

Data collection and data quality assessment. Data of patients with EVD and EBOV RT-PCR results were recorded on a study-specific electronic database. Clinical data were manually collected and eventually crosschecked from clinical records by 2 independent investigators (M. Langer and G. Brogiato). The final assessment of data quality was carried out in a common session (conference call) between EMERGENCY NGO staff in Sierra Leone and INMI staff in Italy.

Laboratory methods. EBOV RNA testing was performed using a qPCR assay (RealStar Filovirus Screen RT-PCR 1.0 kit, Altona Diagnostics), with a LOD of $3.11 \log \mathrm{cp} / \mathrm{ml}$ of EBOV RNA. Viral RNA quantification was based on a standard reference curve provided by the kit producers, spanning up to $9 \log \mathrm{cp} / \mathrm{ml}$ of EBOV RNA.

Malaria testing was performed by BinaxNOW Malaria rapid point-of-care test (Alere).

HIV testing was performed by Alere Determine HIV-1/2 Ag/Ab Combo rapid point-of-care test.

Statistics. Bivariable and multivariable standard linear regression models were used to confirm association between viremia and a patient's clinical outcome, and to select potential confounders to be included in the multilevel regression model. Exposures with fewer than 5 observations were excluded. All covariates with $P \leq 0.100$ in bivariable analyses were included in multivariable analysis. All variables with $P \leq 0.100$ in the multivariable analysis were included in the final multilevel model.

Variation of EBOV viremia over time after symptom onset was modeled according to a polynomial maximum likelihood multilevel linear regression model with random intercept at patient level and random slope at time level (24). These models, historically referred as growthcurve models (25) and eventually referred to latent trajectory models (26), represent a prominent group of mixed-effect linear regression techniques frequently used to analyze repeated measures data taken over time (e.g., clustered longitudinal data). In fact, the flexibility of these models makes it possible to describe linear and nonlinear (polynomial) variation of a continuous dependent variable (in this study, EBOV viremia) over time (in this study, the time after symptom onset). As our study is, in principle, an exploratory research, we preferred the polynomial approach over other relevant inferential techniques, such multilevel spline regression model (27). In fact, we lacked any solid background knowledge about EBOV kinetics in order to define the number and the temporal position of the knots to implement a spline model.

Random intercept and random slope were assessed by LRT. In particular, the random intercept was included if LRT $P$ value for a standard null model (i.e., the model including only the dependent variable) versus the random intercept null model was less than 0.100; random slope was included if LRT $P$ value for the random intercept null model versus the random intercept plus the random slope null model was less than 0.100. The model was implemented with no assumption about variance-covariance structure (unstructured matrix structure for heteroskedastic random effects models).

The functional form of association between EBOV viremia and time after symptom onset (from days 2-13) was decided by LRT to assess subsequent polynomial models with viremia (dependent variable) and time (independent variable) as a continuous variable with increasing exponential power, or as a 12-level categorical variable. In this way, the best functional form for time after symptom onset was chosen according to simplicity and fitness criteria by preferring the most complex model (i.e., that including highest polynomial power) over the simplest one whenever LRT $P \leq 0.100$.

Interaction between patient's outcome (either survivor or nonsurvivor) and time since symptom onset was assessed by LRT. Interaction terms were included for LRT $P$ value $\leq 0.100$.

Model-based punctual estimates of EBOV viremia at different times, difference between survivors and nonsurvivors with relative 95\% CI, and $P$ values were obtained by linear prediction and ANOVAstyle joint tests.

All analyses were carried out by STATA 13.1 statistical package.

Study approval. This work was conducted as part of the international public health response to help the containment of the outbreak in Sierra Leone and, therefore, informed consent was not obtained from individual patients. Ethics approval was obtained from the INMI Lazzaro Spallanzani Ethic Board (protocol registration 307) to use, analyze, and publish unidentified, anonymous data collected during clinical management of patients.

\section{Author contributions}

SL designed the study, analyzed the data, and wrote the manuscript; GP and ML were responsible for patients' clinical management, data collection, and writing the manuscript; GB implemented the onsite database and collected and cross-checked the data entry; FV and $\mathrm{AP}$ contributed to the study design, data cleaning, and writing of the manuscript; CC, MRC, and ADC tested all the samples, designed the laboratory methodology, and contributed to the writing of the manuscript; SK, JA, and RM contributed to data interpretation and manuscript writing; GPK, AZ, GS, and GI contributed ideas for study design and interpretation of the results, and they supervised and contributed to the manuscript writing. All authors read and approved the final manuscript. Members of the INMI-EMERGENCY EBOV Sierra Leone Study group cared for patients, collected samples, and/or carried out diagnostic tests.

\section{Acknowledgments}

Goderich ETC has been built by the Royal Engineers as proxy for the Department for International Development - UK Government (DFID) in cooperation with EMERGENCY's Technical Division. The ETC's operations have been cofunded by DFID and EMERGENCY's private donations. The upgrading of the virology laboratory has been supported by a grant from the Italian Ministry of Foreign Affairs - Direzione General per la Cooperazione allo Sviluppo (DGCS). INMI's research activities have been performed thanks to grants from Italian Ministry of Health (Ricerca Corrente and Ricerca Finalizzata). Deployment of laboratory personnel was possible thanks to a grant from the Italian Ministry of Foreign Affairs - DGCS. We thank the EMlab European consortium (IFS/2011/272372; www.emlab.eu), which has been where we have developed the knowledge and experience that has allowed us to establish the virology laboratory at Goderich ETC. We thank the people who cared for at the ETC in Goderich, Sierra Leone, and all members of the INMI-EMERGENCY EBOV Sierra Leone Study group.

Address correspondence to: Giuseppe Ippolito, INMI Lazzaro Spallanzani Via Portuense, 292-00149 Rome, Italy. Phone: 0039.06.55170.700; E-mail: giuseppe.ippolito@inmi.it. 
1. Gulland A. Institutional failure led to Ebola outbreak "spiralling out of control," says MSF. BMJ. 2015;350:h1619.

2. Lanini S, et al. Are adaptive randomised trials or non-randomised studies the best way to address the Ebola outbreak in West Africa? Lancet Infect Dis. 2015;15(6):738-745.

3. World Health Organization. Ebola Situation Reports. WHO Web site. http://apps.who.int/ ebola/ebola-situation-reports. Updated September 13, 2015. Accessed October 6, 2015.

4. Bah EI, et al. Clinical presentation of patients with Ebola virus disease in Conakry, Guinea. N Engl JMed. 2015;372(1):40-47.

5. Schieffelin JS, et al. Clinical illness and outcomes in patients with Ebola in Sierra Leone. $N$ Engl J Med. 2014;371(22):2092-2100.

6. Cohen-Wolkowiez M, et al. Developmental pharmacokinetics of piperacillin and tazobactam using plasma and dried blood spots from infants. Antimicrob Agents Chemother. 2014;58(5):2856-2865.

7. Ovaskainen O, Soininen J. Making more out of sparse data: hierarchical modeling of species communities. Ecology. 2011;92(2):289-295.

8. McElroy AK, et al. Ebola hemorrhagic fever: novel biomarker correlates of clinical outcome. JInfect Dis. 2014;210(4):558-566.

9. Baize $S$, et al. Defective humoral responses and extensive intravascular apoptosis are associated with fatal outcome in Ebola virus-infected patients. Nat Med.1999;5(4):423-426.

10. McElroy AK, et al. Human Ebola virus infection results in substantial immune activation. Proc Natl Acad Sci U S A. 2015;112(15):4719-4724.

11. Ksiazek TG, et al. Clinical virology of Ebola hemorrhagic fever (EHF): virus, virus antigen, and IgG and IgM antibody findings among EHF patients in Kikwit, Democratic Republic of the Congo, 1995. JInfect Dis. 1999;179(suppl 1):S177-S187.

12. Towner JS, et al. Rapid diagnosis of Ebola hemorrhagic fever by reverse transcription-PCR in an outbreak setting and assessment of patient viral load as a predictor of outcome. JVirol. 2004;78(8):4330-4341.

13. Kortepeter MG, Bausch DG, Bray M. Basic clinical and laboratory features of filoviral hemorrhagic fever. J Infect Dis. 2011;204(suppl 3):S810-S816.

14. How Many Ebola Patients Have Been Treated Outside of Africa? The New York Time Web site. http:// www.nytimes.com/interactive/2014/07/31/world/ africa/ebola-virus-outbreak-qa.html?_r=0. Updated January 26, 2015. Accessed on October 6, 2015.

15. Brouqui P, Ippolito G. Ebola and travel - management of imported cases. Travel Med Infect Dis. 2014;12(6 pt A):561-562.

16. Schnirring L. Health worker critically ill, as Ebola exposures prompt flurry of medical evacuations. Center for Infectious Diseases Research and Policy (CIDRAP) Web site. http://www.cidrap.umn. edu/news-perspective/2015/03/health-workercritically-ill-ebola-exposures-prompt-flurrymedical. Updated March 16, 2015. Accessed on October 6, 2015.

17. Bevilacqua N, et al. Criteria for discharge of patients with Ebola virus diseases in high-income countries [published online ahead of print October 22, 2015]. Lancet Glob Health. http://dx.doi. org/10.1016/S2214-109X(15)00205-3.

18. Liddell AM, et al. Characteristics and clinical management of a cluster of 3 patients with Ebola virus disease, including the first domestically acquired cases in the United States. Ann Intern
Med. 2015;163(2):81-90.

19. Wolf T, et al. Severe Ebola virus disease with vascular leakage and multiorgan failure: treatment of a patient in intensive care. Lancet. 2015;385(9976):1428-1435.

20. Kreuels B, et al. A case of severe Ebola virus infection complicated by gram-negative septicemia. N EnglJMed. 2014;371(25):2394-2401.

21. Grund S, Lüdtke O, Robitzsch A. Multiple imputation of missing covariate values in multilevel models with random slopes: a cautionary note [published online ahead of print May 5, 2015]. Behav Res Methods. doi:10.3758/s13428-015-0590-3.

22. Ebola in Sierra Leone: one year after the first confirmed case. EMERGENCY Web site. http:// www.emergency.it/sierraleone/ebola-one-yearafter-the-first-confirmed-case.html. Updated May 25, 2015. Accessed on October 6, 2015.

23. World Health Organization. Case definition recommendations for Ebola or Marburg virus diseases. WHO Web site. http://www.who.int/ csr/resources/publications/ebola/ebola-casedefinition-contact-en.pdf. Updated August 9, 2014. Accessed on October 6, 2015.

24. Graubard BI, Korn EL. Modelling the sampling design in the analysis of health surveys. Stat Methods Med Res. 1996;5(3):263-281.

25. Goldstein H. Efficient statistical modelling of longitudinal data. Ann Hum Biol. 1986;13(2):129-141.

26. Curran PJ, Hussong AM. The use of latent trajectory models in psychopathology research. J Abnorm Psychol. 2003;112(4):526-544.

27. Edwards LJ, Stewart PW, MacDougall JE, Helms RW. A method for fitting regression splines with varying polynomial order in the linear mixed model. Stat Med. 2006;25(3):513-527. 